\title{
The existence and regularity of multiple solutions for a class of infinitely degenerate elliptic equations *
}

\author{
Hua Chen ${ }^{\dagger} \quad \mathrm{Ke} \mathrm{Li}^{\ddagger}$
}

\begin{abstract}
Let $X=\left(X_{1}, \ldots \ldots, X_{m}\right)$ be an infinitely degenerate system of vector fields, we study the existence and regularity of multiple solutions of Dirichelt problem for a class of semi-linear infinitely degenerate elliptic operators associated with the sum of square operator $\Delta_{X}=\sum_{j=1}^{m} X_{j}^{*} X_{j}$.
\end{abstract}

Keywords: degenerate elliptic equations, Logarithmic Sobolev inequality.

\section{Introduction}

In this paper, we study the existence and regularity of solutions for a class of semi-linear infinitely degenerate elliptic operators. Consider a system of vector fields $X=\left(X_{1}, \ldots \ldots, X_{m}\right)$ defined on an open domain $\widetilde{\Omega} \subset \mathbb{R}^{d}$. We suppose that this system satisfies the following Logarithmic regularity estimates,

$$
\left\|(\log \Lambda)^{s} u\right\|_{L^{2}(\Omega)}^{2} \leq C\left\{\sum_{j=1}^{m}\left\|X_{j} u\right\|_{L^{2}(\Omega)}^{2}+\|u\|_{L^{2}(\Omega)}^{2}\right\}, \forall u \in C_{0}^{\infty}(\widetilde{\Omega}),
$$

where $\Lambda=\left(e^{2}+|D|^{2}\right)^{1 / 2}=\langle D\rangle$. The results of $[4,6,7,8,9]$ gave some sufficient conditions for the estimates (1.1). We remark that if $s>1$, the estimate (1.1) implies the hypoellipticity of the infinitely degenerate elliptic operator $\Delta_{X}=\sum_{j=1}^{m} X_{j}^{*} X_{j}$, where $X_{j}^{*}$ is the formal adjoint of $X_{j}$.

Definition 1.1. If $\Gamma$ is a smooth surfaces of $\widetilde{\Omega}$, we say that $\Gamma$ is non characteristic for the system of vector fields $X$, if for any point $x_{0} \in \Gamma$ there exists at least one vector field in $X=\left(X_{1}, \ldots \ldots X_{m}\right)$ which is transversal to $\Gamma$ at $x_{0}$.

\footnotetext{
* Research supported partially by the NSFC

${ }^{\dagger}$ Department of Mathematics, Wuhan University, 430072-Hubei, China E-mail address: chenhua@whu.edu.cn

${ }^{\ddagger}$ Department of Mathematics, Wuhan University, 430072-Hubei, China. E-mail address: like@zzu.edu.cn
} 
Definition 1.2. Let now $\Gamma=\bigcup_{j \in J} \Gamma_{j}$ be the union of a family of smooth surface in $\widetilde{\Omega}$. We say that $\Gamma$ is non characteristic for $\mathrm{X}$, if for any point $x_{0} \in \Gamma$, there exists at least one vector field of $X_{1}, \ldots, X_{m}$ which is transversal to $\Gamma_{j}$ at $x_{0}$ for all $j$ in which $x_{0} \in \Gamma_{j}$.

We say that the vector fields $X=\left(X_{1}, \ldots \ldots, X_{m}\right)$ satisfies the finite type of Hörmander's condition on an open domain $\omega \subset \widetilde{\Omega}$ in $\mathbb{R}^{d}$ if the rank of the Lie algebra generated by the vector fields $X=\left(X_{1}, \ldots \ldots, X_{m}\right)$ and its finite times commutators is equal to the space dimension $d$ at every point in $\omega$.

A typical example is the vector fields in $\mathbb{R}^{3}$, i.e. $X_{1}=\partial_{x_{1}}, X_{2}=\partial_{x_{2}}, X_{3}=$ $\exp \left(-\left|x_{1}\right|^{-1 / s}\right) \partial_{x_{3}}$ with $s>0$. The operator $\Delta_{X}$ in this example is degenerate infinitely on $\Gamma_{0}=\left\{x_{1}=0\right\}$, and the vector fields $X=\left(X_{1}, X_{2}, X_{3}\right)$ satisfies the finite type of Hörmander's condition in $\mathbb{R}^{3} \backslash \Gamma_{0}$.

The example with infinitely degeneracy on a union of surfaces $\Gamma=\bigcup_{j \in J} \Gamma_{j}$ is the system in $\mathbb{R}^{2}$ such that $X_{1}=\partial_{x_{1}}, X_{2}=\exp \left(-\left(x_{1}^{2} \sin ^{2}\left(\frac{\pi}{x_{1}}\right)\right)^{\frac{-1}{2 s}}\right) \partial_{x_{2}}$, we have $\Gamma_{j}=\left\{x_{1}=\frac{1}{j}\right\}$ for $j \in \mathbb{Z} \backslash\{0\}, \Gamma_{0}=\left\{x_{1}=0\right\}$, then $X_{1}$ is transverse to all $\Gamma_{j}, j \in \mathbb{Z}$, and $X_{2}$ vanishes infinitely on $\Gamma=\bigcup_{j \in \mathbb{Z}} \Gamma_{j}$. The vector fields $X=\left(X_{1}, X_{2}\right)$ satisfies the finite type of Hörmander's condition in $\mathbb{R}^{2} \backslash \Gamma$.

Related to the systems of vector fields $X=\left(X_{1}, \ldots \ldots X_{m}\right)$, Morimoto and $\mathrm{Xu}$ introduce the following function space (cf.[10]),

$$
H_{X}^{1}(\widetilde{\Omega})=\left\{u \in L^{2}(\widetilde{\Omega}), X_{j} u \in L^{2}(\widetilde{\Omega}), j=1, \ldots, m\right\}
$$

which is a Hilbert space with norm $\|u\|_{H_{X}^{1}}^{2}=\|u\|_{L^{2}}^{2}+\|X u\|_{L^{2}}^{2}$, and $\|X u\|_{L^{2}}^{2}=$ $\sum_{j=1}^{m}\left\|X_{j} u\right\|_{L^{2}}^{2}$. Take $\Omega \subset \subset \widetilde{\Omega}$ as a bounded open subset and suppose that $\partial \Omega$ is $C^{\infty}$ and non characteristic for the system of vector fields $X$, Morimoto and Xu define the space $H_{X, 0}^{1}(\Omega)$ as a closure of $C_{0}^{\infty}(\Omega)$ in $H_{X}^{1}(\Omega)$, which is also a Hilbert space.

If the system of vector fields $X$ satisfies the estimates (1.1), we have the following Logarithmic Sobolev inequality;

Proposition 1.1. (cf.[10]) Suppose that the system of vector fields $X=\left(X_{1}, \ldots \ldots, X_{m}\right)$ verifies the estimates (1.1) for some $s>1 / 2$. Then there exists $C_{0}>0$ such that

$$
\int_{\Omega}|v|^{2}\left|\log \left(\frac{|v|}{\|v\|_{L^{2}(\Omega)}}\right)\right|^{2 s-1} \leq C_{0}\left\{\sum_{j=1}^{m}\left\|X_{j} v\right\|_{L^{2}(\Omega)}^{2}+\|v\|_{L^{2}(\Omega)}^{2}\right\},
$$

for all $v \in H_{X, 0}^{1}(\Omega)$. 
Using the Logarithmic Sobolev inequality above, Morimoto and $\mathrm{Xu}$ [10] have studied the following semi-linear Dirichlet problems,

$$
\triangle_{X} u=a u \log |u|+b u,\left.u\right|_{\partial \Omega}=0,
$$

where constant coefficients $a, b \in \mathbb{R}$. They have obtained,

Proposition 1.2. (cf.[10]) We suppose that the system of vector fields $X=\left(X_{1}, \ldots \ldots X_{m}\right)$ satisfies the following conditions:

$\widetilde{H}-1) \partial \Omega$ is $C^{\infty}$ and non characteristic for the system of vector fields $X$;

$\widetilde{H}$-2) the system of vector fields $X$ satisfies the finite type of Hörmander's condition on $\widetilde{\Omega}$ except an union of smooth surfaces $\Gamma$ which are non characteristic for $X$;

$\widetilde{H}-3)$ the system of vector fields $X$ satisfies the estimate (1.1) for $s>3 / 2$.

Suppose $a \neq 0$ in (1.3), then the semi-linear Dirichlet problem (1.3) posses at least one non trivial weak solution $u \in H_{X, 0}^{1}(\Omega) \cap L^{\infty}(\Omega)$. Moreover, if a $>0$, we have $u \in C^{\infty}(\Omega \backslash \Gamma) \bigcap C^{0}(\bar{\Omega} \backslash \Gamma)$ and $u>0$ for all $x \in \Omega \backslash \Gamma$.

Next, it will be useful for us to introduce following Poincarés inequality,

Proposition 1.3. (cf.[10]) Under the hypotheses $\widetilde{H}-1), \widetilde{H}-2)$ and $\widetilde{H}-3)$, the first eigenvalue $\lambda_{1}$ of the operator $\triangle_{X}$ is strictly positive, which is equivalent to following Poincarés s inequality

$$
\|\varphi\|_{L^{2}}^{2} \leq \frac{1}{\lambda_{1}}\|X \varphi\|_{L^{2}}^{2}, \quad \forall \varphi \in H_{X, 0}^{1}(\Omega) .
$$

In this paper, we shall study the following semi-linear Dirichlet problem

$$
\begin{aligned}
-\triangle_{X} u & =a(x) u \log |u|+b(x) u+g(x), \quad \text { in } \Omega, \\
\left.u\right|_{\partial \Omega} & =0 .
\end{aligned}
$$

Our main result is as follows.

Theorem 1.4. Suppose that the system of vector fields $X=\left(X_{1}, \ldots \ldots X_{m}\right)$ satisfies the following conditions:

$H-1) \partial \Omega$ is $C^{\infty}$ and non characteristic for the system of vector fields $X$;

$H-2)$ the system of vector fields $X$ satisfies the finite type of Hörmander's condition on $\widetilde{\Omega}$ except an union of smooth surfaces $\Gamma$ which are non characteristic for $X$;

$H$-3) the system of vector fields $X$ satisfies the estimate (1.1) for $s \geq 5 / 2$;

$H$-4) $a(x), b(x) \in L^{\infty}(\Omega)$, and there exist $a_{0}, b_{0} \in \mathbb{R}_{+}$, such that $a(x) \geq a_{0}$, and $b(x) \geq$ $b_{0}$, a.e. in $\Omega$. Then 
1) there exists $C>0$ such that the problem (1.5) and (1.6) has at least two solutions in $H_{X, 0}^{1}(\Omega)$, for any $g \not \equiv 0$ satisfying $\|g\|_{\left.L^{2}(\Omega)\right)}<C$;

2) the problem (1.5) and (1.6) has at least one non-negative solution $u \in H_{X, 0}^{1}(\Omega)$; furthermore, if $g(x) \in L^{\infty}(\Omega)$, then the non-negative solution $u(x) \in L^{\infty}(\Omega)$.

3) If $a(x), b(x), g(x) \in C^{\infty}(\Omega)$, and there exists $g_{0}>0$ such that $g(x) \geq g_{0}$, then we have $u \in C^{\infty}(\Omega \backslash \Gamma) \bigcap C^{0}(\bar{\Omega} \backslash \Gamma)$ and $u(x)>0$ for all $x \in \Omega \backslash \Gamma$.

The proof of Theorem 1.4 relies essentially on the Ekeland Variational Principle (cf.[5]) and on the Mountain Pass Theorem without the Palais-Smale condition, established by Brezis-Nirenberg [3], namely

Proposition 1.5. (cf.[5]) Let $V$ be a complete metric space, and $F: V \rightarrow \mathbb{R} \cup\{+\infty\}$ a lower semicontinuous function, $\not \equiv+\infty$, bounded from below. For any $\epsilon>0$, there is some point $v \in V$ with

$$
\begin{gathered}
F(v) \leq \inf _{V} F+\epsilon . \\
\forall w \in V, F(w) \geq F(v)-\epsilon d(v, w) .
\end{gathered}
$$

Proposition 1.6. (cf.[3]) Let $\Phi$ be a $C^{1}$ function on a Banach space E. Suppose there exists a neighborhood $U$ of 0 in $E$ and a constant $\rho$ such that $\Phi(u) \geq \rho$ for every $u$ in the boundary of $U$,

$$
\Phi(0)<\rho \text {, and } \Phi(v)<\rho \text { for some } v \notin U \text {. }
$$

Set

$$
c=\inf _{\mathbb{P} \in M} \max _{W \in \mathbb{P}} \Phi(w) \geq \rho,
$$

where $M$ denotes the class of paths joining 0 to $v$.

Conclusion: there is a sequence $\left\{u_{i}\right\}$ in $E$ such that

$$
\Phi\left(u_{i}\right) \rightarrow c \text { and } \Phi^{\prime}\left(u_{i}\right) \rightarrow 0 \text { in } E^{*}
$$

\section{Auxiliary results}

Definition 2.1. We say that $u \in H_{X, 0}^{1}(\Omega)$ is a weak solution of (1.5) and (1.6) if

$$
\int_{\Omega} \sum_{j=1}^{m} X_{j} u X_{j} v d x-\int_{\Omega} a(x) u v \log |u| d x-\int_{\Omega} b(x) u v d x-\int_{\Omega} g(x) v d x=0
$$

for all $v \in C_{0}^{\infty}(\Omega)$. 
We define the function $J_{\eta}, H_{X, 0}^{1}(\Omega) \rightarrow \mathbb{R}, 0 \leq \eta<1$ by

$$
\begin{aligned}
J_{\eta}(u) & =\int_{\Omega} \sum_{j=1}^{m}\left(X_{j} u\right)^{2} d x-\int_{\Omega} a(x) u^{2} \log (|u|+\eta) d x+\int_{\Omega} \frac{a(x) u^{2}|u|}{2(|u|+\eta)} \\
& -\int_{\Omega} b(x) u^{2} d x-2 \int_{\Omega} g(x) u d x .
\end{aligned}
$$

A simple calculation shows that as $0<\eta<1, J_{\eta} \in C^{1}\left(H_{X, 0}^{1}(\Omega), \mathbb{R}\right)$ and it's derivative is given by,

$$
\begin{aligned}
\left\langle J_{\eta}^{\prime}(u), v\right\rangle & =2 \int_{\Omega} \sum_{j=1}^{m}\left(X_{j} u\right)\left(X_{j} v\right)-2 \int_{\Omega} a(x) u v \log (|u|+\eta) d x \\
& +\int_{\Omega} \frac{a(x) u|u| v \eta}{2(|u|+\eta)^{2}} d x-2 \int_{\Omega} b(x) u v d x-2 \int_{\Omega} g(x) v d x,
\end{aligned}
$$

for all $u, v \in H_{X, 0}^{1}(\Omega)$.

We have denoted by $\langle\cdot, \cdot\rangle$ the duality pairing between $H_{X, 0}^{1}(\Omega)$ and $H_{X, 0}^{-1}(\Omega)$, and $H_{X, 0}^{-1}(\Omega)$ is the dual space of $H_{X, 0}^{1}(\Omega)$, i.e. $H_{X, 0}^{-1}(\Omega)=\left(H_{X, 0}^{1}(\Omega)\right)^{*}$. We use the notation $\rightarrow$ as the weak convergence and the notation $\rightarrow$ as the strong convergence in Banach space.

Definition 2.2. If $F$ is a $C^{1}$ functional on some Banach space $\mathrm{E}$ and $c$ is a real number, we say that a sequence $\left\{u_{n}\right\}$ in $\mathrm{E}$ is a $(P S)_{c}$ sequence of $F$ if $F\left(u_{n}\right) \rightarrow c$ and $F^{\prime}\left(u_{n}\right) \rightarrow 0$ in $E^{*}$.

Remark: If $\left\{u_{n}\right\}$ is a bounded sequence in $H_{X, 0}^{1}(\Omega)$, then there exists a subsequence (denote still by $\left\{u_{n}\right\}$ ) such that $u_{n} \rightarrow u_{0}$ in $H_{X, 0}^{1}(\Omega), u_{n} \rightarrow u_{0}$ in $L^{2}(\Omega)$.

Lemma 2.1. Let $M>0$ and let $\left\{v_{j}, j \in \mathbb{N}\right\}$ be a sequence in $H_{X, 0}^{1}(\Omega)$, satisfying

$$
\left\|v_{j}\right\|_{H_{X, 0}^{1}(\Omega)}^{2} \leq M
$$

Then $\left\{\left|v_{j}\right||\log | v_{j}||\right\}$ is uniformly integrable.

\section{Proof.}

$$
\begin{aligned}
& \left.\int_{\Omega}\left|v_{j}\right||\log | v_{j}\right|^{2} \leq \frac{1}{2}|\Omega|+\left.\frac{1}{2} \int_{\Omega} v_{j}^{2}|\log | v_{j}\right|^{4} d x \\
= & \frac{1}{2}|\Omega|+\frac{1}{2} \int_{\Omega} v_{j}^{2}\left|\log \frac{\left|v_{j}\right|}{\left\|v_{j}\right\|_{L^{2}}}+\log \left\|v_{j}\right\|_{L^{2}}\right|^{4} d x \\
\leq & \frac{1}{2}|\Omega|+4 \int_{\Omega} v_{j}^{2} \log ^{4} \frac{\left|v_{j}\right|}{\left\|v_{j}\right\|_{L^{2}}}+4\left|\log \left\|v_{j}\right\|_{L^{2}}\right|^{4}\left\|v_{j}\right\|_{L^{2}}^{2} \\
\leq & \frac{1}{2}|\Omega|+4 C_{0}\left(\left\|X v_{j}\right\|_{L^{2}}^{2}+\left\|v_{j}\right\|_{L^{2}}^{2}\right)+4\left|\log \left\|v_{j}\right\|_{L^{2}}\right|^{4}\left\|v_{j}\right\|_{L^{2}}^{2}
\end{aligned}
$$




$$
\begin{aligned}
& =\frac{1}{2}|\Omega|+4 C_{0}\left(\left\|X v_{j}\right\|_{L^{2}}^{2}+\left\|v_{j}\right\|_{L^{2}}^{2}\right)+\frac{4}{2^{4}}\left|\log \left\|v_{j}\right\|_{L^{2}}^{2}\right|^{4}\left\|v_{j}\right\|_{L^{2}}^{2} \\
& \leq \frac{1}{2}|\Omega|+4 C_{0} M+\frac{4}{2^{4}}\left[\left(4 e^{-1}\right)^{4}+(\log M)^{4} M\right] \\
& =\tilde{M}
\end{aligned}
$$

where $C_{0}>0$ is a positive constant given by Proposition 1.1. We use the fact $t(\log t)^{4} \leq l \log ^{4} l+\left(4 e^{-1}\right)^{4}$ for any $0 \leq t \leq l$.

Now, we prove that for any $\epsilon>0$, there exists $\delta>0$ such that if $A \subset \Omega$, the measure of $A, \mu(A)<\delta$, then

$$
\int_{A}\left|v_{j}\right||\log | v_{j}||<\epsilon, \quad \forall j .
$$

But for any $\epsilon>0$, there exists $t_{0}>e^{2}$ such that

$$
\frac{1}{\log t}<\epsilon, \quad \forall t \geq t_{0} .
$$

Take now $\delta=\epsilon\left(t_{0} \log t_{0}\right)^{-1}, \mu(A)<\delta$ and

$$
A_{j}=A \cap\left\{\left|v_{j}\right| \leq t_{0}\right\}, \quad B_{j}=A \cap\left\{\left|v_{j}\right|>t_{0}\right\},
$$

then we have,

$$
\begin{array}{r}
\int_{A_{j}}\left|v_{j}\right||\log | v_{j}|| \leq t_{0} \log t_{0} \mu\left(A_{j}\right)<\epsilon, \\
\int_{B_{j}}\left|v_{j}\right||\log | v_{j}|| \leq \epsilon \int_{B_{j}}\left|v_{j}\right||\log | v_{j}||^{2}<\epsilon \tilde{M} .
\end{array}
$$

The proof of Lemma 2.1 is complete.

Lemma 2.2. If $a(x) \in L^{\infty}(\Omega), \zeta \in C_{0}^{\infty}(\Omega)$, $\left\|u_{n}\right\|_{H_{X, 0}^{1}(\Omega)}<M, M$ is a positive constant independent of $n$, then there exists a convergent subsequence (denote still by $\left.\left\{u_{n}\right\}\right)$ such that $u_{n} \rightarrow u_{0}$ in $H_{X, 0}^{1}(\Omega)$ and

$$
\lim _{n \rightarrow \infty} \int_{\Omega} a(x) u_{n} \zeta \log \left(\left|u_{n}\right|+1 / 2^{n}\right) d x=\int_{\Omega} a(x) u_{0} \zeta \log \left(\left|u_{0}\right|\right) d x .
$$

Proof. We have

$$
\begin{aligned}
& \int_{\Omega}\left|a(x) u_{n} \zeta\right|\left|\log \left(\left|u_{n}\right|+2^{-n}\right)\right|^{2} d x \leq C \int_{\Omega}\left|u_{n}\right|\left|\log \left(\left|u_{n}\right|+2^{-n}\right)\right|^{2} d x \\
\leq & C \int_{\left\{x:\left|u_{n}\right|+2^{-n} \leq 1\right\}}\left|u_{n}\right|\left|\log \left(\left|u_{n}\right|+2^{-n}\right)\right|^{2} d x \\
+ & C \int_{\left\{x:\left|u_{n}\right|+2^{-n} \geq 1\right\}}\left|u_{n}\right|\left|\log \left(\left|u_{n}\right|+2^{-n}\right)\right|^{2} d x
\end{aligned}
$$




$$
\begin{aligned}
& \leq C \int_{\left\{x:\left|u_{n}\right|+2^{-n} \leq 1\right\}}\left|u_{n}\right|\left|\log \left(\left|u_{n}\right|\right)\right|^{2} d x \\
& +C \int_{\left\{x:\left|u_{n}\right|+2^{-n} \geq 1\right\}}\left|u_{n}\right|\left|\log \left(2\left|u_{n}\right|\right)\right|^{2} d x \\
& \leq C \int_{\left\{x:\left|u_{n}\right|+2^{-n} \leq 1\right\}}\left|u_{n}\right|\left|\log \left(\left|u_{n}\right|\right)\right|^{2} d x \\
& +C \int_{\left\{x:\left|u_{n}\right|+2^{-n} \geq 1\right\}}\left|u_{n}\right|\left(\log ^{2} 2+\left|\log \left(\left|u_{n}\right|\right)\right|^{2}\right) d x \\
& \leq C \int_{\Omega}\left|u_{n}\right|\left|\log \left(\left|u_{n}\right|\right)\right|^{2} d x+C\left(\int_{\Omega}\left|u_{n}\right|^{2} d x+|\Omega|\right),
\end{aligned}
$$

since $a(x) \in L^{\infty}(\Omega), \zeta \in C_{0}^{\infty}(\Omega)$. By the proof of Lemma 2.1, we know there exists $\tilde{M}$, such that

$$
\int_{\Omega}\left|a(x) u_{n} \xi\right|\left|\log \left(\left|u_{n}\right|+2^{-n}\right)\right|^{2} d x \leq \tilde{M} .
$$

Next, we prove that for any $\epsilon>0$, there exists $\delta>0$ such that if $A \subset \Omega, \mu(A)<\delta$, then

$$
\int_{A}\left|a(x) u_{n} \zeta\right|\left|\log \left(\left|u_{n}\right|+2^{-n}\right)\right| d x<\epsilon, \quad \forall n .
$$

But for any $\epsilon>0$, there exists $t_{0}>e^{2}$ such that

$$
\frac{1}{\log t}<\epsilon, \quad \forall t \geq t_{0}
$$

Take now $\delta=\epsilon\left\{a_{\infty} \max _{x \in \Omega}|\zeta(x)|\left[\left(t_{0}+2^{-1}\right)^{2}+e^{-1}\right]\right\}^{-1}, \mu(A)<\delta, a_{\infty}=\|a(x)\|_{L^{\infty}(\Omega)}$ and

$$
A_{n}=A \cap\left\{\left|u_{n}\right| \leq t_{0}\right\}, \quad B_{n}=A \cap\left\{\left|u_{n}\right|>t_{0}\right\},
$$

then we have,

$$
\begin{aligned}
& \int_{A_{n}}\left|a(x) u_{n} \zeta\right|\left|\log \left(\left|u_{n}\right|+2^{-n}\right)\right| d x \\
& \leq a_{\infty} \max _{x \in \Omega}|\zeta(x)| \int_{A_{n}}\left|u_{n}\right|\left|\log \left(\left|u_{n}\right|+2^{-n}\right)\right| d x \\
& \leq a_{\infty} \max _{x \in \Omega}|\zeta(x)| \int_{A_{n}}\left[\left(\left|u_{n}\right|+2^{-n}\right)^{2}+e^{-1}\right] \\
& \leq a_{\infty} \max _{x \in \Omega}|\zeta(x)|\left[\left(\left|t_{0}\right|+2^{-1}\right)^{2}+e^{-1}\right] \mu\left(A_{n}\right) \\
&< \epsilon, \\
& \int_{B_{n}}\left|a(x) u_{n} \zeta\right|\left|\log \left(\left|u_{n}\right|+2^{-n}\right)\right| d x<\epsilon \int_{B_{n}}\left|a(x) u_{n} \zeta\right|\left|\log \left(\left|u_{n}\right|+2^{-n}\right)\right|^{2} d x<\epsilon \tilde{M} .
\end{aligned}
$$

Similarly, we can prove that 
Lemma 2.3. For any fixed $0<\eta<<1, a(x) \in L^{\infty}(\Omega), \zeta \in C_{0}^{\infty}(\Omega),\left\|u_{n}\right\|_{H_{X, 0}^{1}(\Omega)}<$ $M, M$ is a positive constant independent of $n$, there exists a convergent subsequence (denote still by $\left\{u_{n}\right\}$ ) such that $u_{n} \rightarrow u_{0}$ in $H_{X, 0}^{1}(\Omega)$, and

$$
\lim _{n \rightarrow \infty} \int_{\Omega} a(x) u_{n} \zeta \log \left(\left|u_{n}\right|+\eta\right) d x=\int_{\Omega} a(x) u_{0} \zeta \log \left(\left|u_{0}\right|+\eta\right) d x
$$

Lemma 2.4. For any fixed $0<\eta<<1, a(x) \in L^{\infty}(\Omega), u(x) \in H_{X, 0}^{1}(\Omega), u_{n} \in C_{0}^{\infty}(\Omega)$ and $\left\|u_{n}-u\right\|_{H_{X, 0}^{1}(\Omega)} \rightarrow 0$, we have

$$
\lim _{n \rightarrow \infty} \int_{\Omega} a(x) u u_{n} \log \left(\left|u_{n}\right|+\eta\right) d x=\int_{\Omega} a(x) u^{2} \log (|u|+\eta) d x .
$$

Lemma 2.5. If $a(x) \in L^{\infty}(\Omega), u(x) \in H_{X, 0}^{1}(\Omega), u_{n} \in C_{0}^{\infty}(\Omega)$ and $\left\|u_{n}-u\right\|_{H_{X, 0}^{1}(\Omega)} \rightarrow$ 0 , we have

$$
\lim _{n \rightarrow \infty} \int_{\Omega} a(x) u u_{n} \log \left(\left|u_{n}\right|\right) d x=\int_{\Omega} a(x) u^{2} \log (|u|) d x .
$$

Similar to Lemma 2.1, we have

Lemma 2.6. Let $M>0$ and let $\left\{v_{j}, j \in \mathbb{N}\right\}$ be a sequence in $H_{X, 0}^{1}(\Omega)$ satisfying

$$
\left\|v_{j}\right\|_{H_{X, 0}^{1}(\Omega)}^{2} \leq M
$$

Then there exists a convergent sub-sequence $\left\{v_{j_{k}}\right\}$ such that $v_{j_{k}} \rightarrow v_{0} \in H_{X, 0}^{1}(\Omega)$ and

$$
\lim _{j_{k} \rightarrow \infty} \int_{\Omega}\left|v_{j_{k}}\right|^{2}|\log | v_{j_{k}}||=\int_{\Omega}\left|v_{0}\right|^{2}|\log | v_{0}||
$$

and

$$
\int_{\Omega}\left|v_{0}\right|^{2}|\log | v_{0}|| \leq C M
$$

where $C$ is a positive constant independent of $j$.

Proof. Using the fact $|t \log t| \leq t^{2}+e^{-1}$, for $\forall t>0$, we have

$$
\begin{aligned}
\int_{\Omega}\left|v_{j}\right|^{2}|\log | v_{j} \|^{2} & =\int_{\Omega}\left|v_{j}\right|^{2}\left|\log \frac{\left|v_{j}\right|}{\left\|v_{j}\right\|_{L^{2}}}+\log \left\|v_{j}\right\|_{L^{2}}\right|^{2} \\
& \leq 2 \int_{\Omega}\left|v_{j}\right|^{2}\left|\log \frac{\left|v_{j}\right|}{\left\|v_{j}\right\|_{L^{2}}}\right|^{2}+2\left\|v_{j}\right\|_{L^{2}(\Omega)}^{2}\left|\log \left\|v_{j}\right\|_{L^{2}}\right|^{2} \\
& \leq 2 C_{0}\left(\left\|X v_{j}\right\|_{L^{2}}^{2}+\left\|v_{j}\right\|_{L^{2}}^{2}\right)+2\left(M+e^{-1}\right)^{2} \\
& \leq 2 C_{0} M+4\left(M^{2}+e^{-2}\right) \\
& =\tilde{M}
\end{aligned}
$$


$C_{0}$ is a positive constant given by Proposition 1.1. The rest of the proof is similar to the proof of Lemma 2.1.

Next, we can prove that for any $\epsilon>0$, there exists $\delta>0$, such that if $A \subset$ $\Omega, \mu(A)<\delta$, then

$$
\int_{A}\left|v_{j}\right|^{2}|\log | v_{j}|| d x<\epsilon, \quad \forall j
$$

Actually for any $\epsilon>0$, there exists $t_{0}>e^{2}$, such that

$$
\frac{1}{\log t}<\epsilon, \quad \forall t \geq t_{0}
$$

Take now $\delta=\epsilon\left(t_{0}^{2} \log t_{0}+\frac{1}{2} e^{-1}\right)^{-1}, \mu(A)<\delta$ and

$$
A_{j}=A \cap\left\{\left|v_{j}\right| \leq t_{0}\right\}, \quad B_{j}=A \cap\left\{\left|v_{j}\right|>t_{0}\right\},
$$

then we have,

$$
\begin{gathered}
\int_{A_{j}}\left|v_{j}\right|^{2}|\log | v_{j}|| d x \leq \int_{A_{j}}\left(t_{0}^{2} \log t_{0}+\frac{1}{2} e^{-1}\right)<\left(t_{0}^{2} \log t_{0}+\frac{1}{2} e^{-1}\right) \mu\left(A_{j}\right)<\epsilon, \\
\int_{B_{j}}\left|v_{j}\right|^{2}|\log | v_{j}|| d x \leq \epsilon \int_{B_{j}}\left|v_{j}\right|^{2}|\log | v_{j}||^{2} d x<\epsilon \tilde{M} .
\end{gathered}
$$

Thus we have

Lemma 2.7. For any fixed $0<\eta<<1, a(x) \in L^{\infty}(\Omega), u_{n} \in H_{X, 0}^{1}(\Omega)$ and $\left\|u_{n}\right\|_{H_{X, 0}^{1}(\Omega)}<$ $M,(M$ is a positive constant independent of $n)$ there exists a convergent subsequence (denote still by $\left\{u_{n}\right\}$ ) such that $u_{n} \rightarrow u_{0}$ in $H_{X, 0}^{1}(\Omega)$ and

$$
\lim _{n \rightarrow \infty} \int_{\Omega} a(x) u_{n} u_{0} \log \left(\left|u_{n}\right|+\eta\right) d x=\int_{\Omega} a(x) u_{0}^{2} \log \left(\left|u_{0}\right|+\eta\right) d x .
$$

Lemma 2.8. If $a(x) \in L^{\infty}(\Omega),\left\|u_{n}\right\|_{H_{X, 0}^{1}(\Omega)}<M, M$ is a positive constant independent of $n$, then there exists a convergent subsequence (denote still by $\left\{u_{n}\right\}$ ) such that $u_{n} \rightarrow u_{0}$ in $H_{X, 0}^{1}(\Omega)$ and

$$
\lim _{n \rightarrow \infty} \int_{\Omega} a(x) u_{n}^{2} \log \left(\left|u_{n}\right|+1 / 2^{n}\right) d x=\int_{\Omega} a(x) u_{0}^{2} \log \left(\left|u_{0}\right|\right) d x
$$




\section{The existence of solutions}

For any fixed $0<\epsilon<1,0<\eta<<1$ and $u \in H_{X, 0}^{1}(\Omega)$, by using Young's inequality, Proposition 1.1 and Proposition 1.3, we have,

$$
\begin{aligned}
& J_{\eta}(u)=\|X u\|_{L^{2}(\Omega)}^{2}-\int_{\Omega} a(x) u^{2} \log (|u|+\eta) d x+\int_{\Omega} \frac{a(x) u^{2}|u|}{2(|u|+\eta)}-\int_{\Omega} b(x) u^{2} d x \\
& -2 \int_{\Omega} g(x) u d x \\
& =\|X u\|_{L^{2}(\Omega)}^{2}-\int_{|u|>\eta} a(x) u^{2} \log (|u|+\eta) d x-\int_{|u| \leq \eta} a(x) u^{2} \log (|u|+\eta) d x \\
& +\int_{\Omega} \frac{a(x) u^{2}|u|}{2(|u|+\eta)}-\int_{\Omega} b(x) u^{2} d x-2 \int_{\Omega} g(x) u d x \\
& \geq\|X u\|_{L^{2}(\Omega)}^{2}-\int_{|u|>\eta} a(x) u^{2} \log 2|u| d x-\log 2 \eta \int_{|u| \leq \eta} a(x) u^{2} d x \\
& +\int_{\Omega} \frac{a(x) u^{2}|u|}{2(|u|+\eta)}-\int_{\Omega} b(x) u^{2} d x-2 \int_{\Omega} g(x) u d x \\
& \geq\|X u\|_{L^{2}(\Omega)}^{2}-\log 2 \int_{|u|>\eta} a(x) u^{2} d x-\int_{|u|>\eta} a(x) u^{2}\left(\log \frac{|u|}{\|u\|_{L^{2}}}+\log \|u\|_{L^{2}}\right) d x \\
& -a_{0} \log 2 \eta \int_{|u| \leq \eta} u^{2} d x+\int_{\Omega} \frac{a(x) u^{2}|u|}{2(|u|+\eta)}-\int_{\Omega} b(x) u^{2} d x-2 \int_{\Omega} g(x) u d x \\
& >\|X u\|_{L^{2}(\Omega)}^{2}-a_{\infty} \log 2 \int_{\Omega} u^{2} d x-\frac{\epsilon}{C_{0}} \int_{\Omega} u^{2} \log ^{2} \frac{|u|}{\|u\|_{L^{2}}}-\frac{C_{0}}{4 \epsilon} \int_{\Omega} a^{2}(x) u^{2} \\
& -\log \|u\|_{L^{2}} \int_{|u|>\eta} a(x) u^{2}-a_{0} \log 2 \eta \int_{|u| \leq \eta} u^{2} d x+\int_{\Omega} \frac{a(x) u^{2}|u|}{2(|u|+\eta)} \\
& -b_{\infty} \int_{\Omega} u^{2} d x-\int_{\Omega} g^{2}(x) d x-\int_{\Omega} u^{2}(x) d x \\
& >\|X u\|_{L^{2}(\Omega)}^{2}-a_{\infty} \log 2 \int_{\Omega} u^{2} d x-\epsilon\left(\|X u\|_{L^{2}}^{2}+\|u\|_{L^{2}}^{2}\right)-\frac{C_{0} a_{\infty}^{2}}{4 \epsilon} \int_{\Omega} u^{2} \\
& -\log \|u\|_{L^{2}} \int_{|u|>\eta} a(x) u^{2}-a_{0} \log 2 \eta \int_{|u| \leq \eta} u^{2} d x+\int_{\Omega} \frac{a(x) u^{2}|u|}{2(|u|+\eta)} \\
& -b_{\infty} \int_{\Omega} u^{2} d x-\int_{\Omega} g^{2}(x) d x-\int_{\Omega} u^{2}(x) d x \\
& >(1-\epsilon) \frac{\lambda_{1}}{1+\lambda_{1}}\|u\|_{H_{X, 0}^{1}(\Omega)}^{2}-C_{1}\|u\|_{L^{2}(\Omega)}^{2}-\log \|u\|_{L^{2}} \int_{|u|>\eta} a(x) u^{2} d x \\
& -a_{0} \log 2 \eta \int_{|u| \leq \eta} u^{2} d x+\int_{\Omega} \frac{a(x) u^{2}|u|}{2(|u|+\eta)}-\|g\|_{L^{2}(\Omega)}^{2}
\end{aligned}
$$




$$
\begin{aligned}
& >(1-\epsilon) \frac{\lambda_{1}}{1+\lambda_{1}}\|u\|_{H_{X, 0}^{1}(\Omega)}^{2}-C_{1}\|u\|_{L^{2}(\Omega)}^{2}-\log \|u\|_{H_{X, 0}^{1}(\Omega)} \int_{|u|>\eta} a(x) u^{2} d x \\
& -a_{0} \log 2 \eta \int_{|u| \leq \eta} u^{2} d x+\int_{\Omega} \frac{a(x) u^{2}|u|}{2(|u|+\eta)}-\|g\|_{L^{2}(\Omega)}^{2},
\end{aligned}
$$

where $C_{1}=a_{\infty} \log 2+\epsilon+\frac{C_{0}}{4 \epsilon} a_{\infty}^{2}+b_{\infty}+1, C_{0}>0$ is a positive constant given by Proposition 1.1, $a_{\infty}=\|a\|_{L^{\infty}}, b_{\infty}=\|b\|_{L^{\infty}}$.

If we set $B_{R}=\left\{u \in H_{X, 0}^{1}(\Omega),\|u\|_{H_{X, 0}^{1}(\Omega)}<R\right\}$, the estimate above shows that, as $\eta$ is small enough, there exist $R=R(\epsilon)>0$, and $\delta=\delta(R)>0$ such that $\left.J_{\eta}(u)\right|_{\partial B_{R}} \geq \delta>0$ for all $g$ with $\|g\|_{L^{2}(\Omega)} \leq C$. For example, we can take,

$$
\begin{gathered}
R(\epsilon)=\exp \left\{\frac{C_{1}}{-a_{0}}\right\}, \quad C=C(\epsilon)=\frac{R}{2} \sqrt{\frac{\lambda_{1}(1-\epsilon)}{1+\lambda_{1}}}, \\
\delta(R)=\frac{\lambda_{1}(1-\epsilon)}{8\left(1+\lambda_{1}\right)} R^{2}(\epsilon), \quad \eta<\frac{1}{2} \exp \left\{\frac{C_{1}}{-a_{0}}\right\} .
\end{gathered}
$$

Define $c_{\eta}=c_{\eta}(R)=\inf _{u \in \bar{B}_{R}} J_{\eta}(u)$, then $c_{\eta} \leq J_{\eta}(0)=0$. The set $\bar{B}_{R}$ becomes a complete metric space with respect to the distance,

$$
\operatorname{dist}(u, v)=\|u-v\|_{H_{X, 0}^{1}(\Omega)} \text { for any } u, v \in \bar{B}_{R} .
$$

On the other hand, $J_{\eta}$ is lower semi-continuous and bounded from below on $\bar{B}_{R}$. So, by Proposition 1.5 (cf. [5] Theorem 1.1), for any positive integer $n$ there exists $\left\{u_{\eta, n}\right\}$, satisfying

$$
\begin{gathered}
c_{\eta} \leq J_{\eta}\left(u_{\eta, n}\right) \leq c_{\eta}+\frac{1}{n} \\
J_{\eta}(w) \geq J_{\eta}\left(u_{\eta, n}\right)-\frac{1}{n}\left\|u_{\eta, n}-w\right\|_{H_{X, 0}^{1}(\Omega)} \text { for all } w \in \bar{B}_{R} .
\end{gathered}
$$

We claim that $0<\left\|u_{\eta, n}\right\|_{H_{X, 0}^{1}(\Omega)}<R$ for any $n$ large enough. Indeed, if $\left\|u_{\eta, n}\right\|_{H_{X, 0}^{1}(\Omega)}=R$ for infinitely many $n$, we may assume, without loss of generality, that $\left\|u_{\eta, n}\right\|_{H_{X, 0}^{1}(\Omega)}=R$ for all $n \geq 1$. It follows that $J_{\eta}\left(u_{\eta, n}\right) \geq \delta>0$. Combining this with (3.1) and letting $n \rightarrow \infty$, we have $0 \geq c_{\eta} \geq \delta>0$ which is a contradiction.

We now prove that $J_{\eta}^{\prime}\left(u_{\eta, n}\right) \rightarrow 0$ as $n \rightarrow \infty$ in $H_{X, 0}^{-1}(\Omega)$. Indeed, for any $u \in$ $H_{X, 0}^{-1}(\Omega)$ with $\|u\|_{H_{X, 0}^{1}(\Omega)}=1$, let $w_{n}=u_{\eta, n}+t u$. For a fixed $n$, we have $\left\|w_{n}\right\|_{H_{X, 0}^{1}(\Omega)} \leq$ $\left\|u_{\eta, n}\right\|_{H_{X, 0}^{1}(\Omega)}+t<R$, where $t>0$ is small enough. From (3.2) we obtain

$$
J_{\eta}\left(u_{\eta, n}+t u\right) \geq J_{\eta}\left(u_{\eta, n}\right)-\frac{t}{n}\|u\|_{H_{X, 0}^{1}(\Omega)}
$$

that is

$$
\frac{J_{\eta}\left(u_{\eta, n}+t u\right)-J_{\eta}\left(u_{\eta, n}\right)}{t} \geq-\frac{1}{n}\|u\|_{H_{X, 0}^{1}(\Omega)}=-\frac{1}{n} .
$$


Letting $t \searrow 0$, we deduce that $\left\langle J_{\eta}^{\prime}\left(u_{\eta, n}\right), u\right\rangle \geq-1 / n$ and a similar argument for $t \nearrow 0$ produces $\left|\left\langle J_{\eta}^{\prime}\left(u_{\eta, n}\right), u\right\rangle\right| \leq 1 / n$ for any $u \in H_{X, 0}^{1}(\Omega)$ with $\|u\|_{H_{X, 0}^{1}(\Omega)}=1$. So

$$
\left\|J_{\eta}^{\prime}\left(u_{\eta, n}\right)\right\|_{-1}=\sup _{\substack{u \in H_{X, 0}^{1}(\Omega) \\\|u\|_{H_{X, 0}^{1}(\Omega)}^{1}=1}}\left|\left\langle J_{\eta}^{\prime}\left(u_{\eta, n}\right), u\right\rangle\right| \leq \frac{1}{n} \rightarrow 0 \text { as } n \rightarrow \infty .
$$

Thus, $\left\{u_{\eta, n}\right\}$ is a $(P S)_{c_{\eta}}$ sequence in $H_{X, 0}^{1}(\Omega)$, i.e.

$$
J_{\eta}\left(u_{\eta, n}\right) \rightarrow c_{\eta} \text {, and } J_{\eta}^{\prime}\left(u_{\eta, n}\right) \rightarrow 0 \text { in } H_{X, 0}^{-1}(\Omega) .
$$

Since $\left\|u_{\eta, n}\right\|_{H_{X, 0}^{1}(\Omega)} \leq R,\left\{u_{\eta, n}\right\}$ is a bounded sequence in $H_{X, 0}^{1}(\Omega)$, and passing to a subsequence (denote still by $\left\{u_{\eta, n}\right\}$ ), we may assume that $u_{\eta, n} \rightarrow u_{\eta, 0}$ in $H_{X, 0}^{1}(\Omega)$ for some $u_{\eta, 0} \in H_{X, 0}^{1}(\Omega)$. So, by Lemma 2.3 , we know that $J_{\eta}^{\prime}\left(u_{\eta, 0}\right)=0$, i.e.

$$
\begin{aligned}
2 \int_{\Omega} \sum_{j=1}^{m}\left(X_{j} u_{\eta, 0}\right)\left(X_{j} v\right) & -2 \int_{\Omega} a(x) u_{\eta, 0} v \log \left(\left|u_{\eta, 0}\right|+\eta\right) d x+\int_{\Omega} \frac{a(x) u_{\eta, 0}^{2} v \eta}{2\left(\left|u_{\eta, 0}\right|+\eta\right)^{2}} d x \\
& -2 \int_{\Omega} b(x) u_{\eta, 0} v d x-2 \int_{\Omega} g(x) v d x=0
\end{aligned}
$$

for all $v \in C_{0}^{\infty}(\Omega)$.

We know $\left\{u_{\eta, 0}\right\}$ is also bounded in $H_{X, 0}^{1}(\Omega)$. For $\eta=\eta_{i}=\frac{1}{2^{i}}, \frac{1}{2^{i}}<\frac{1}{2} \exp \left\{\frac{C_{1}}{-a_{0}}\right\}$, passing to a subsequence (denote still by $\left\{u_{\eta, n}\right\}$ ), we may assume that $u_{\eta_{i}, 0} \rightarrow u_{0}$ in $H_{X, 0}^{1}(\Omega)$ as $i \rightarrow \infty$. Now by Lemma 2.2 , we have,

$$
\int_{\Omega} \sum_{j=1}^{m}\left(X_{j} u_{0}\right)\left(X_{j} v\right)-\int_{\Omega} a(x) u_{0} v \log \left|u_{0}\right| d x-\int_{\Omega} b(x) u_{0} v d x-\int_{\Omega} g(x) v d x=0
$$

$u_{0}$ is a weak solution of (1.5) and (1.6).

We can prove that $J_{0}\left(u_{0}\right)=c_{0}$. Actually, we have

$$
\begin{aligned}
& J_{\eta}\left(u_{\eta, n}\right)+\frac{1}{2}\left\|J_{\eta}^{\prime}\left(u_{\eta, n}\right)\right\|_{-1}\left\|u_{\eta, n}\right\|_{H_{X, 0}^{1}(\Omega)} \geq J_{\eta}\left(u_{\eta, n}\right)-\frac{1}{2}<J_{\eta}^{\prime}\left(u_{\eta, n}\right), u_{\eta, n}> \\
= & \int_{\Omega} \frac{a(x) u_{\eta, n}^{2}\left|u_{\eta, n}\right|}{2\left(\left|u_{\eta, n}\right|+\eta\right)}-\int_{\Omega} \frac{a(x) u_{\eta, n}^{2}\left|u_{\eta, n}\right| \eta}{4\left(\left|u_{\eta, n}\right|+\eta\right)^{2}}-\int_{\Omega} g u_{\eta, n} .
\end{aligned}
$$

Letting $n \rightarrow \infty$, we know

$$
c_{\eta} \geq \int_{\Omega} \frac{a(x) u_{\eta, 0}^{2}\left|u_{\eta, 0}\right|}{2\left(\left|u_{\eta, 0}\right|+\eta\right)}-\int_{\Omega} \frac{a(x) u_{\eta, 0}^{2}\left|u_{\eta, 0}\right| \eta}{4\left(\left|u_{\eta, 0}\right|+\eta\right)^{2}}-\int_{\Omega} g u_{\eta, 0} .
$$

By Lemma 2.7, we have

$$
\begin{aligned}
0 & =\left\langle J_{\eta_{i}}^{\prime}\left(u_{\eta_{i}, 0}\right), u_{\eta_{i}, 0}\right\rangle=2\left\|X u_{\eta_{i}, 0}\right\|_{L^{2}}^{2}-2 \int_{\Omega} a(x) u_{\eta_{i}, 0}^{2} \log \left(\left|u_{\eta_{i}, 0}\right|+\eta_{i}\right) d x \\
& +\int_{\Omega} \frac{a(x) u_{\eta_{i}, 0}^{2}\left|u_{\eta_{i}, 0}\right| \eta_{i}}{2\left(\left|u_{\eta_{i}, 0}\right|+\eta_{i}\right)^{2}} d x-2 \int_{\Omega} b(x) u_{\eta_{i}, 0}^{2} d x-2 \int_{\Omega} g(x) u_{\eta_{i}, 0} d x
\end{aligned}
$$


Therefore

$$
\begin{aligned}
J_{\eta_{i}}\left(u_{\eta_{i}, 0}\right) & =\int_{\Omega} \frac{a(x) u_{\eta_{i}, 0}^{2}\left|u_{\eta_{i}, 0}\right|}{2\left(\left|u_{\eta_{i}, 0}\right|+\eta_{i}\right)} d x-\int_{\Omega} \frac{a(x) u_{\eta_{i}, 0}^{2}\left|u_{\eta_{i}, 0}\right| \eta_{i}}{4\left(\left|u_{\eta_{i}, 0}\right|+\eta_{i}\right)^{2}} d x \\
& -\int_{\Omega} g(x) u_{\eta_{i}, 0} d x .
\end{aligned}
$$

By (3.5), (3.6) and (3.7), we have:

$$
\begin{aligned}
0 \geq c_{0} & =\inf _{u \in \bar{B}_{R}} J_{0}(u) \geq \lim _{i \rightarrow \infty} \inf _{u \in \bar{B}_{R}} J_{\eta_{i}}(u)=\lim _{i \rightarrow \infty} c_{\eta_{i}} \\
& \geq \frac{1}{2} \int_{\Omega} a(x) u_{0}^{2} d x-\int_{\Omega} g(x) u_{0} d x=J_{0}\left(u_{0}\right) .
\end{aligned}
$$

Since $u_{0} \in \bar{B}_{R}$, it follows that $J_{0}\left(u_{0}\right)=c_{0}$.

On the other hand, letting $\tilde{u} \in H_{X, 0}^{1}(\Omega),\|\tilde{u}\|_{H_{X, 0}^{1}(\Omega)}=R$, and $t>0$, we have

$$
\begin{aligned}
J_{\eta}(t \tilde{u}) & <J_{0}(t \tilde{u})=t^{2}\left[\|X \tilde{u}\|_{L^{2}(\Omega)}^{2}-\log t \int_{\Omega} a(x) \tilde{u}^{2}-\int_{\Omega} a(x) \tilde{u}^{2} \log |\tilde{u}|\right. \\
& \left.+\frac{1}{2} \int_{\Omega} a(x) \tilde{u}^{2}-\int_{\Omega} b(x) \tilde{u}^{2}-2 \int_{\Omega} g(x) \tilde{u} / t\right] \\
& <t^{2}\left[\|X \tilde{u}\|_{L^{2}(\Omega)}^{2}-\log t \int_{\Omega} a(x) \tilde{u}^{2}-\int_{\Omega} a(x) \tilde{u}^{2} \log |\tilde{u}|\right. \\
& \left.+\frac{1}{2} \int_{\Omega} a(x) \tilde{u}^{2}-\int_{\Omega} b(x) \tilde{u}^{2}+\frac{1}{t}\left(\int_{\Omega} g^{2}(x)+\int_{\Omega} \tilde{u}^{2}\right)\right]
\end{aligned}
$$

We can find $\bar{t}>>1$, such that $J_{\eta}(t \tilde{u})<J_{0}(t \tilde{u})<0$ for all $t \geq \bar{t}$. Letting $\bar{u}=\bar{t} \tilde{u}$, then we have $\|\bar{u}\|_{H_{X, 0}^{1}(\Omega)}>R$ and $J_{\eta}(\bar{u})<0$.

We put

$$
\begin{gathered}
\varrho=\left\{\gamma \in C\left([0,1], H_{X, 0}^{1}(\Omega)\right): \gamma(0)=0, \gamma(1)=\bar{t} \tilde{u},\right\}, \\
\bar{c}_{\eta}=\inf _{\gamma \in \varrho} \sup _{u \in \gamma} J_{\eta}(u) .
\end{gathered}
$$

For $\gamma_{0}=\{t \bar{t} \tilde{u}: 0 \leq t \leq 1\}$, we have

$$
\begin{aligned}
& \sup _{u \in \gamma_{0}} J_{\eta}(u) \leq \sup _{u \in \gamma_{0}} J_{0}(u)=\sup _{0 \leq t \leq 1}\left[(t \bar{t})^{2}\|X \tilde{u}\|_{L^{2}(\Omega)}^{2}-(t \bar{t})^{2} \log (t \bar{t}) \int_{\Omega} a(x) \tilde{u}^{2}\right. \\
- & \left.(t \bar{t})^{2} \int_{\Omega} a(x) \tilde{u}^{2} \log |\tilde{u}|+\frac{(t \bar{t})^{2}}{2} \int_{\Omega} a(x) \tilde{u}^{2}-(t \bar{t})^{2} \int_{\Omega} b(x) \tilde{u}^{2}-2(t \bar{t}) \int_{\Omega} g(x) \tilde{u}\right] \\
\leq & \bar{t}^{2}\|X \tilde{u}\|_{L^{2}(\Omega)}^{2}+\frac{1}{2 e} \int_{\Omega} a(x) \tilde{u}^{2}+\bar{t}^{2} \int_{\Omega} a(x) \tilde{u}^{2}|\log | \tilde{u} \|+\frac{\bar{t}^{2}}{2} \int_{\Omega} a(x) \tilde{u}^{2} \\
+ & \bar{t} \int_{\Omega} g^{2}+\bar{t} \int_{\Omega} \tilde{u}^{2} .
\end{aligned}
$$


So there exists a positive constant $B$ (which is independent of $\eta$ ), satisfying

$$
\bar{c}_{\eta} \leq B
$$

It follows from the Proposition 1.6 (cf. [3] Theorem 2.2) that there is a $(P S)_{c_{\eta}}$ sequence $\left\{u_{\eta, n}\right\}$ of $J_{\eta}(u)$ such that

$$
J_{\eta}\left(u_{\eta, n}\right)=\bar{c}_{\eta}+o(1) \text { and } J_{\eta}^{\prime}\left(u_{\eta, n}\right) \rightarrow 0 \quad \text { in } \quad H_{X, 0}^{-1}(\Omega) .
$$

We have

$$
\begin{aligned}
& J_{\eta}(u)-\frac{1}{2}<J_{\eta}^{\prime}(u), u>=\int_{\Omega} \frac{a(x) u^{2}|u|}{2(|u|+\eta)} d x-\int_{\Omega} \frac{a(x) u^{2}|u| \eta}{4(|u|+\eta)^{2}} d x-\int_{\Omega} g(x) u d x \\
> & \int_{\Omega} \frac{a(x) u^{2}|u|}{4(|u|+\eta)} d x-\frac{a_{0}}{16} \int_{\Omega} u^{2} d x-\frac{4}{a_{0}} \int_{\Omega} g^{2} d x \\
= & \int_{|u|>\eta} \frac{a(x) u^{2}|u|}{4(|u|+\eta)} d x+\int_{|u| \leq \eta} \frac{a(x) u^{2}|u|}{4(|u|+\eta)} d x-\frac{a_{0}}{16} \int_{|u|>\eta} u^{2} d x-\frac{a_{0}}{16} \int_{|u| \leq \eta} u^{2} d x \\
- & \frac{4}{a_{0}} \int_{\Omega} g^{2} d x \\
> & \frac{1}{4} \int_{|u|>\eta} \frac{a(x) u^{2}|u|}{2|u|} d x-\frac{a_{0}}{16} \int_{|u|>\eta} u^{2} d x-\frac{a_{0}}{16} \int_{|u| \leq \eta} u^{2} d x-\frac{4}{a_{0}} \int_{\Omega} g^{2} d x \\
> & \frac{a_{0}}{16} \int_{|u|>\eta} u^{2} d x-\frac{a_{0} \eta^{2}|\Omega|}{16}-\frac{4}{a_{0}}\|g\|_{L^{2}}^{2} .
\end{aligned}
$$

So, we have

$$
\begin{aligned}
& \bar{c}_{\eta}+o(1)+\frac{1}{2}\left\|J_{\eta}^{\prime}\left(u_{\eta, n}\right)\right\|_{-1}\left\|u_{\eta, n}\right\|_{H_{X, 0}^{1}(\Omega)}+\frac{a_{0}|\Omega|}{16}+\frac{4}{a_{0}}\|g\|_{L^{2}}^{2} \\
\geq & J_{\eta}\left(u_{\eta, n}\right)-\frac{1}{2}\left\langle J_{\eta}^{\prime}\left(u_{\eta, n}\right), u_{\eta, n}\right\rangle+\frac{a_{0}|\Omega|}{16}+\frac{4}{a_{0}}\|g\|_{L^{2}}^{2} \\
> & \frac{a_{0}}{16} \int_{|u|>\eta} u_{\eta, n}^{2} d x .
\end{aligned}
$$

By (3.10), we have

$$
\begin{aligned}
& \int_{\Omega}\left|u_{\eta, n}\right|^{2} d x=\int_{|u|>\eta}\left|u_{\eta, n}\right|^{2} d x+\int_{u \leq \eta}\left|u_{\eta, n}\right|^{2} d x \\
< & \frac{16}{a_{0}}\left[\bar{c}_{\eta}+o(1)+\frac{1}{2}\left\|J_{\eta}^{\prime}\left(u_{\eta, n}\right)\right\|_{-1}\left\|u_{\eta, n}\right\|_{H_{X, 0}^{1}(\Omega)}++\frac{a_{0}|\Omega|}{16}+\frac{4}{a_{0}}\|g\|_{L^{2}}^{2}\right]+\eta^{2}|\Omega| \\
< & \frac{16}{a_{0}}\left[B+o(1)+\frac{1}{2}\left\|J_{\eta}^{\prime}\left(u_{\eta, n}\right)\right\|_{-1}\left\|u_{\eta, n}\right\|_{H_{X, 0}^{1}(\Omega)}+\frac{a_{0}|\Omega|}{16}+\frac{4}{a_{0}}\|g\|_{L^{2}}^{2}\right]+|\Omega| \\
< & C+C\left\|J_{\eta}^{\prime}\left(u_{\eta, n}\right)\right\|_{-1}\left\|u_{\eta, n}\right\|_{H_{X, 0}^{1}(\Omega)}+o(1),
\end{aligned}
$$


where $C$ is a positive constant which is independent of $\eta$ and $n$, and dependent of $|\Omega|,\|g\|_{L^{2}}^{2}, a_{0}$, and $B$. Similar to the estimate of $J_{\eta}(u)$ at the beginning of this section, we have (if taking $\epsilon=\frac{1}{2}$ )

$$
\begin{aligned}
B+o(1) & >\bar{c}_{\eta}+o(1)=J_{\eta}\left(u_{\eta, n}\right) \geq \frac{\lambda_{1}}{2\left(1+\lambda_{1}\right)}\left\|u_{\eta, n}\right\|_{H_{X, 0}^{1}}^{2}-C_{1}\left\|u_{\eta, n}\right\|_{L^{2}}^{2} \\
& -a_{\infty}\left\|u_{\eta, n}\right\|_{L^{2}}^{2}\left|\log \left\|u_{\eta, n}\right\|_{L^{2}}\right|-\|g\|_{L^{2}(\Omega)}^{2}
\end{aligned}
$$

where $C_{1}=a_{\infty} \log 2+\frac{C_{0}}{2} a_{\infty}^{2}+b_{\infty}+\frac{3}{2}$, to be independent of $\eta$ and $n$, and $C_{0}$ and $\lambda_{1}$ are given by Proposition 1.1 and Proposition 1.3 respectively.

Furthermore, using the fact $|t \log t| \leq t^{2}+e^{-1}$ for $t \geq 0$, we have

$$
\begin{aligned}
\frac{\lambda_{1}}{2\left(1+\lambda_{1}\right)}\left\|u_{\eta, n}\right\|_{H_{X, 0}^{1}}^{2} & \leq B+o(1)+C_{1}\left\|u_{\eta, n}\right\|_{L^{2}}^{2}+a_{\infty}\left|\left\|u_{\eta, n}\right\|_{L^{2}}^{2}\right| \log \left\|u_{\eta, n}\right\|_{L^{2}} \mid+\|g\|_{L^{2}(\Omega)}^{2} \\
& \leq B+o(1)+C_{1}\left\|u_{\eta, n}\right\|_{L^{2}}^{2}+\frac{1}{2} a_{\infty}\left(\left\|u_{\eta, n}\right\|_{L^{2}}^{4} \mid+e^{-1}\right)+\|g\|_{L^{2}(\Omega)}^{2} \\
& <C+o(1)+C\left\|u_{\eta, n}\right\|_{L^{2}}^{2}+C\left\|u_{\eta, n}\right\|_{L^{2}}^{4},
\end{aligned}
$$

where $C$ is independent of $\eta$ and $n$.

By (3.11), we have

$\left\|u_{\eta, n}\right\|_{H_{X, 0}^{1}}^{2} \leq C+o(1)+C\left\|J_{\eta}^{\prime}\left(u_{\eta, n}\right)\right\|_{-1}\left\|u_{\eta, n}\right\|_{H_{X, 0}^{1}(\Omega)}+C\left\|J_{\eta}^{\prime}\left(u_{\eta, n}\right)\right\|_{-1}^{2}\left\|u_{\eta, n}\right\|_{H_{X, 0}^{1}(\Omega)}^{2}$.

Since $J_{\eta}^{\prime}\left(u_{\eta, n}\right) \rightarrow 0$ in $H_{X, 0}^{-1}(\Omega)$, thus there exists $N_{0}>0$ such that $\left\|u_{\eta, n}\right\|_{H_{X, 0}^{1}}^{2} \leq$ $M$, if $n>N_{0}$, where $M$ is a constant, independent of $\eta$ and $n$. That means $\left\{u_{\eta}, N_{0}+j\right\}_{j \in N}$ is a bounded sequence in $H_{X, 0}^{1}(\Omega)$. Hence there exists a subsequence (we still denote by $\left\{u_{\eta, n}\right\}$ ), such that $u_{\eta, n} \rightarrow u_{\eta, 0}$ in $H_{X, 0}^{1}(\Omega)$ for some $u_{\eta, 0} \in H_{X, 0}^{1}(\Omega)$. By Lemma 2.3, we have $J_{\eta}^{\prime}\left(u_{\eta, 0}\right)=0$, that is

$$
\begin{gathered}
2 \int_{\Omega} \sum_{j=1}^{m}\left(X_{j} u_{\eta, 0}\right)\left(X_{j} v\right)-2 \int_{\Omega} a(x) u_{\eta, 0} v \log \left(\left|u_{\eta, 0}\right|+\eta\right) d x \\
+\int_{\Omega} \frac{a(x) u_{\eta, 0}\left|u_{\eta, 0}\right| v \eta}{2\left(u_{\eta, 0}+\eta\right)^{2}} d x-2 \int_{\Omega} b(x) u_{\eta, 0} v d x-2 \int_{\Omega} g(x) v d x=0,
\end{gathered}
$$

for any $v \in C_{0}^{\infty}(\Omega)$.

For $\eta=\eta_{i}=\frac{1}{2^{i}}, \frac{1}{2^{i}}<\frac{1}{2} \exp \left\{\frac{C_{1}}{-a_{0}}\right\}$, we know $\left\{u_{\eta_{i}, 0}\right\}$ is also bounded in $H_{X, 0}^{1}(\Omega)$. Passing to a subsequence, we may assume that $u_{\eta_{i}, 0} \rightarrow u_{1}$ in $H_{X, 0}^{1}(\Omega)$ as $i \rightarrow \infty$. Now by Lemma 2.2, we have,

$$
\begin{aligned}
& \int_{\Omega} \sum_{j=1}^{m}\left(X_{j} u_{1}\right)\left(X_{j} v\right)-\int_{\Omega} a(x) u_{1} v \log \left|u_{1}\right| d x-\int_{\Omega} b(x) u_{1} v d x \\
- & \int_{\Omega} g(x) v d x=0
\end{aligned}
$$


for all $v \in C_{0}^{\infty}(\Omega)$. That means $u_{1}$ is a weak solution of problem (1.5) and (1.6).

Next, we prove $u_{\eta_{i}, 0} \rightarrow u_{1}$ in $H_{X, 0}^{1}(\Omega)$. In fact, $C_{0}^{\infty}(\Omega)$ is dense in $H_{X, 0}^{1}(\Omega)$, thus from Lemma 2.4 and Lemma 2.5, we know that (3.12) and (3.13) are also true for any $v \in H_{X, 0}^{1}(\Omega)$.

Especially, we have

$$
\begin{gathered}
2 \int_{\Omega} \sum_{j=1}^{m}\left(X_{j} u_{\eta_{i}, 0}\right)^{2}-2 \int_{\Omega} a(x) u_{\eta_{i}, 0}^{2} \log \left(\left|u_{\eta_{i}, 0}\right|+\eta_{i}\right) d x \\
+\int_{\Omega} \frac{a(x) u_{\eta_{i}, 0}^{2}\left|u_{\eta_{i}, 0}\right| \eta_{i}}{2\left(\left|u_{\eta_{i}, 0}\right|+\eta_{i}\right)^{2}} d x-2 \int_{\Omega} b(x) u_{\eta_{i}, 0}^{2} d x-2 \int_{\Omega} g(x) u_{\eta_{i}, 0} d x=0 \\
\int_{\Omega} \sum_{j=1}^{m}\left(X_{j} u_{1}\right)^{2}-\int_{\Omega} a(x) u_{1}^{2} \log \left|u_{1}\right| d x-\int_{\Omega} b(x) u_{1}^{2} d x-\int_{\Omega} g(x) u_{1} d x=0 .
\end{gathered}
$$

Letting $i \rightarrow \infty$ in (3.14), and from Lemma 2.8 and (3.15), we have

$$
\left\|X_{j} u_{\eta_{i}, 0}\right\|_{L^{2}(\Omega)} \rightarrow\left\|X_{j} u_{1}\right\|_{L^{2}(\Omega)}, \quad i \rightarrow \infty
$$

which means $u_{\eta_{i}, 0} \rightarrow u_{1}$ in $H_{X, 0}^{1}(\Omega)$.

Now by Proposition $1.6([3])$, we have

$$
J_{0}\left(u_{1}\right)=\lim _{i \rightarrow \infty} J_{\eta_{i}}\left(u_{\eta_{i}, 0}\right)=\bar{c}_{0}>0 \geq J_{0}\left(u_{0}\right),
$$

that means the problem (1.5) and (1.6) has at least two solutions in $H_{X, 0}^{1}(\Omega)$.

If we replace, at the beginning, $B_{R}$ by $B_{R}^{+}=\left\{u \in H_{X, 0}^{1}(\Omega),\|u\|_{H_{X, 0}^{1}(\Omega)}<R, u \geq\right.$ $0\}$, thus it is similar to the proof of existence of the solution $u_{0}$, we can deduce that the problem (1.5) and (1.6) has a non-negative solution in $H_{X, 0}^{1}(\Omega)$.

\section{Boundedness and regularity of weak solutions}

Similar to the proof of [10], we can deduce the boundedness and regularity of weak solutions.

By using the interpolation inequality, the condition H-3) and the Logarithmic Sobolev inequality (1.2) give that, for any $N \geq 1$, there exists $C_{N}$ such that,

$$
\int_{\Omega} v^{2} \log ^{2}\left(\frac{|v|}{\|v\|_{L^{2}}}\right) \leq \frac{1}{N}\|X v\|_{L^{2}}^{2}+C_{N}\|v\|_{L^{2}}^{2},
$$

for all $v \in H_{X, 0}^{1}(\Omega)$. 
In order to prove that the solution $u \in L^{\infty}(\Omega)$, it suffices to show that, under the assumptions of Theorem 1.4, there exists $\bar{A}>0$ such that the estimate

$$
\|u\|_{L^{p}} \leq \bar{A}
$$

holds for any $p \geq 2$. In fact, for $\epsilon>0, \Omega_{\epsilon}=\{x \in \Omega ;|u(x)| \geq \bar{A}+\epsilon\}$, it follows from (4.2) that $\left|\Omega_{\epsilon}\right| \leq\left(\frac{\bar{A}}{\bar{A}+\epsilon}\right)^{p} \rightarrow 0($ as $p \rightarrow \infty)$ and hence we have $\|u\|_{L^{\infty}} \leq \bar{A}$.

We prove the estimate (4.2) by the following three steps. First, for any $p \geq 1$, $m \in \mathbf{N}$, we shall use $u^{2 p-1}$ or $u^{2 p-1} \log ^{2 m}\left(u^{p}\right)$ as test function for the equation (1.5). Since we do not know if $u^{2 p-1} \log ^{2 m}\left(u^{p}\right) \in H_{X, 0}^{1}(\Omega)$, so we replace the function $u$ by $u_{(k)}$, where $k>1$ and $u_{(k)}(x)=u(x)$ if $x \in\{x \in \Omega ;|u(x)|<k\}$ and $u_{(k)}(x)=k$ if $x \in\{x \in \Omega ;|u(x)| \geq k\}$. Then it is easy to check (see [6] and [7, Theorem 7 and Theorem 8]) that $u_{(k)}^{2 p-1} \log ^{2 m}\left(u_{(k)}^{p}\right) \in H_{X, 0}^{1}(\Omega)$ for all $p>1, m \in \mathbb{N}$. In the case of $p=1$, we use $u\left(\log ^{m} u\right)_{(k)}^{2} \in H_{X, 0}^{1}(\Omega)$ as the test function. To simplify the notation, we shall drop the subscript and use $u^{2 p-1} \log ^{2 m}\left(u^{p}\right)$ as the test function. We have

Proposition 4.1. Under the hypotheses H-1), H-2), H-3), H-4) of Theorem 1.4, and $g(x) \in L^{\infty}(\Omega), u \in H_{X, 0}^{1}(\Omega), u \geq 0,\|u\|_{L^{2}(\Omega)} \neq 0$ be a weak solution of the equation (1.5). Suppose that for some $p_{0} \geq 1$, there exists $A_{0}, A_{1}$ such that

$$
0<A_{1} \leq\|u\|_{L^{2 p_{0}}} \leq A_{0}
$$

Then

$$
\begin{aligned}
& \int_{\Omega}\left|X(\bar{u})^{p_{0}}\right|^{2}+\int_{\Omega}(\bar{u})^{2 p_{0}} \log ^{2}\left(\bar{u}^{p_{0}}\right) \\
\leq & 2 C_{2}+a_{\infty}^{2}+2 p_{0}\left[b_{\infty}+a_{\infty}\left|\log A_{0}\right|+(1+|\Omega|) g_{\infty} / A_{1}\right],
\end{aligned}
$$

where $a_{\infty}=\|a\|_{L^{\infty}}, b_{\infty}=\|b\|_{L^{\infty}}, g_{\infty}=\|g\|_{L^{\infty}}$ and the constant $C_{2}$ is given by (4.1) and $\bar{u}=u /\|u\|_{L^{2 p_{0}}}$.

Proof. We have $\bar{u} \in H_{X, 0}^{1}(\Omega),\|\bar{u}\|_{L^{2 p_{0}}}=1$, and $\bar{u}$ is a weak solution of equation

$$
-\triangle_{X} \bar{u}=a(x) \bar{u} \log \bar{u}+\left(a(x) \log \|u\|_{L^{2 p_{0}}}+b(x)\right) \bar{u}+\frac{g(x)}{\|u\|_{L^{2 p_{0}}}} .
$$

Take $\bar{u}^{2 p_{0}-1}$ as the test function, we have

$$
\begin{aligned}
& \frac{2 p_{0}-1}{p_{0}^{2}} \int_{\Omega}\left|X \bar{u}^{p_{0}}\right|^{2}=\frac{1}{p_{0}} \int_{\Omega} a(x) \bar{u}^{2 p_{0}} \log \bar{u}^{p_{0}} \\
+ & \int_{\Omega}\left(a(x) \log \|u\|_{L^{2 p_{0}}}+b(x)\right) \bar{u}^{2 p_{0}}+\frac{1}{\|u\|_{L^{2 p_{0}}}} \int_{\Omega} g(x) \bar{u}^{2 p_{0}-1},
\end{aligned}
$$


where

$$
\begin{aligned}
& \frac{1}{\|u\|_{L^{2 p_{0}}}} \int_{\Omega} g(x) \bar{u}^{2 p_{0}-1} \leq \frac{g_{\infty}}{A_{1}}\left[\int_{\bar{u}>1}\left|\bar{u}^{2 p_{0}-1}\right|+\int_{\bar{u} \leq 1}\left|\bar{u}^{2 p_{0}-1}\right|\right] \\
\leq & \frac{g_{\infty}}{A_{1}}\left(\int_{\bar{u}>1} \bar{u}^{2 p_{0}}+|\Omega|\right) \leq \frac{g_{\infty}}{A_{1}}\left(\int_{\Omega} \bar{u}^{2 p_{0}}+|\Omega|\right)=\frac{(1+|\Omega|) g_{\infty}}{A_{1}} .
\end{aligned}
$$

Furthermore

$$
\int_{\Omega}\left|X \bar{u}^{p_{0}}\right|^{2} \leq \frac{1}{2} \int_{\Omega} \bar{u}^{2 p_{0}} \log ^{2}\left(\bar{u}^{p_{0}}\right)+\frac{1}{2} a_{\infty}^{2}+p_{0} a_{\infty}\left|\log A_{0}\right|+p_{0} b_{\infty}+\frac{(1+|\Omega|) p_{0} g_{\infty}}{A_{1}} .
$$

On the other hand, the Logarithmic Sobolev inequality (4.1) gives

$$
\int_{\Omega}\left(u^{p_{0}}\right)^{2} \log ^{2}\left(\frac{\left|u^{p_{0}}\right|}{\left\|u^{p_{0}}\right\|_{L^{2}}}\right) \leq \frac{1}{2}\left\|X\left(u^{p_{0}}\right)\right\|_{L^{2}}^{2}+C_{2}\left\|u^{p_{0}}\right\|_{L^{2}}^{2} .
$$

Note that $\left\|u^{p_{0}}\right\|_{L^{2}}=\|u\|_{L^{2 p_{0}}}^{p_{0}}$ and $\bar{u}=u /\|u\|_{L^{2 p_{0}}}$, we have

$$
\int_{\Omega} \bar{u}^{2 p_{0}} \log ^{2}\left(\bar{u}^{p_{0}}\right) \leq \frac{1}{2}\left\|X\left(\bar{u}^{p_{0}}\right)\right\|_{L^{2}}^{2}+C_{2} .
$$

Adding (4.5) and (4.6), we have the desired estimate (4.3).

Proposition 4.2. We have for any $m \in \mathbb{N}$,

$$
\int_{\Omega}\left|X\left(\bar{u}^{p_{0}}\right)\right|^{2} \log ^{2 m-2}\left(\bar{u}^{p_{0}}\right)+\int_{\Omega} \bar{u}^{2 p_{0}} \log ^{2 m}\left(\bar{u}^{p_{0}}\right) \leq M_{1}^{2 m} P\left(m, p_{0}\right)(m !)^{2}
$$

where $P\left(m, p_{0}\right)=p_{0}^{m}$ if $m \leq \sqrt{p_{0}}, \quad P\left(m, p_{0}\right)=p_{0}^{\sqrt{p_{0}}}$ if $m>\sqrt{p_{0}}$, and

$$
M_{1} \geq\left(2|\Omega|+4 C_{2}+2 C_{4}+10+6 a_{\infty}^{2}+8 b_{\infty}+8 a_{\infty}\left|\log A_{0}\right|+4 g_{\infty}(1+|\Omega|) / A_{1}\right)^{\frac{1}{2}} .
$$

Proof. From the estimate $0<A_{1} \leq\|u\|_{L^{2 p_{0}}} \leq A_{0}$, we have the estimate (4.7) for $m=1$. By induction, we suppose that (4.7) is also hold for $m \in \mathbb{N}$, then we need to prove that (4.7) is hold for $m+1$. Here we simplify the notation again, i.e. $\bar{u}$ and $p_{0}$ would be replaced by $u$ and $p$ in the equation (4.4). We take $u^{2 p-1} \log ^{2 m}\left(u^{p}\right)$ as the test function in (4.4), then

$$
\begin{aligned}
& \frac{2 p-1}{p^{2}} \int_{\Omega}\left|X u^{p}\right|^{2} \log ^{2 m}\left(u^{p}\right)+\frac{2 m}{p} \int_{\Omega}\left|X u^{p}\right|^{2} \log ^{2 m-1}\left(u^{p}\right) \\
= & \frac{1}{p} \int_{\Omega} a(x) u^{2 p} \log ^{2 m+1}\left(u^{p}\right)+\int_{\Omega}\left(a(x) \log \|u\|_{L^{2 p}}+b(x)\right) u^{2 p} \log ^{2 m}\left(u^{p}\right) \\
+ & \int_{\Omega} \frac{g(x)}{\|u\|_{L^{2 p}}} u^{2 p-1} \log ^{2 m}\left(u^{p}\right) .
\end{aligned}
$$


That is

$$
\begin{aligned}
& \int_{\Omega}\left|X u^{p}\right|^{2} \log ^{2 m}\left(u^{p}\right) \leq \frac{1}{2} \int_{\Omega}\left|X u^{p}\right|^{2} \log ^{2 m}\left(u^{p}\right)+2 m^{2} \int_{\Omega}\left|X u^{p}\right|^{2} \log ^{2 m-2}\left(u^{p}\right) \\
+ & \frac{1}{4} \int_{\Omega} u^{2 p} \log ^{2 m+2}\left(u^{p}\right)+\left(a_{\infty}^{2}+p a_{\infty} \log A_{0}+p b_{\infty}\right) \int_{\Omega} u^{2 p} \log ^{2 m}\left(u^{p}\right) \\
+ & \frac{p g_{\infty}}{A_{1}} \int_{\Omega} u^{2 p-1} \log ^{2 m}\left(u^{p}\right) .
\end{aligned}
$$

Using the fact $l^{l} \leq e^{l} l$, we have

$$
\begin{aligned}
& \int_{\Omega} u^{2 p-1} \log ^{2 m}\left(u^{p}\right)=\int_{|u|<1} u^{2 p-1} \log ^{2 m}\left(u^{p}\right)+\int_{|u| \geq 1} u^{2 p-1} \log ^{2 m}\left(u^{p}\right) \\
\leq & 2^{2 m}(m !)^{2}|\Omega|+\int_{\Omega} u^{2 p} \log ^{2 m}\left(u^{p}\right)<(1+|\Omega|) M_{1}^{2 m} P(m, p)(m !)^{2},
\end{aligned}
$$

so that

$$
\begin{aligned}
& \int_{\Omega}\left|X u^{p}\right|^{2} \log ^{2 m}\left(u^{p}\right) \leq \frac{1}{2} \int_{\Omega}\left(u^{p}\right)^{2} \log ^{2 m+2}\left(u^{p}\right)+\left[4 m^{2}+2 a_{\infty}^{2}+\right. \\
& \left.2\left(p a_{\infty}\left|\log A_{0}\right|+p b_{\infty}+p g_{\infty}(1+|\Omega|) / A_{1}\right)\right] M_{1}^{2 m} P(m, p)(m !)^{2} .
\end{aligned}
$$

We study now the term $\int_{\Omega} u^{2 p} \log ^{2 m+2}\left(u^{p}\right)$. Set $\Omega=\Omega_{1} \bigcup \Omega_{2}^{+} \bigcup \Omega_{2}^{-}$with $\Omega_{1}=$ $\{x \in \Omega ; u(x) \leq 1\}$ and

$$
\begin{aligned}
& \Omega_{2}^{+}=\left\{x \in \Omega ; \quad u(x)>1, \quad\left|\log ^{m}\left(u^{p}\right)\right| \leq\left\|u^{p} \log ^{m}\left(u^{p}\right)\right\|_{L^{2}}\right\}, \\
& \Omega_{2}^{-}=\left\{x \in \Omega ; \quad u(x)>1, \quad\left|\log ^{m}\left(u^{p}\right)\right|>\left\|u^{p} \log ^{m}\left(u^{p}\right)\right\|_{L^{2}}\right\} .
\end{aligned}
$$

Then

$$
\int_{\Omega_{1}} u^{2 p} \log ^{2 m+2}\left(u^{p}\right) \leq|\Omega|((m+1) !)^{2} .
$$

For the second part, (4.3) gives

$$
\begin{aligned}
& \int_{\Omega_{2}^{+}} u^{2 p} \log ^{2 m+2}\left(u^{p}\right) \leq\left\|u^{p} \log ^{m}\left(u^{p}\right)\right\|_{L^{2}}^{2} \int_{\Omega} u^{2 p} \log ^{2}\left(u^{p}\right) \\
\leq & \left(2 C_{2}+a_{\infty}^{2}+2 p b_{\infty}+2 p a_{\infty}\left|\log A_{0}\right|+(1+|\Omega|) g_{\infty} / A_{1}\right) M_{1}^{2 m} P(m, p)(m !)^{2} .
\end{aligned}
$$

Next, for the third part, we use the Logarithmic Sobolev inequality (4.1) for $N=4$,

$$
\begin{aligned}
\int_{\Omega_{2}^{-}} u^{2 p} \log ^{2 m+2}\left(u^{p}\right) & \leq \int_{\Omega_{2}^{-}}\left(u^{p} \log ^{m} u^{p}\right)^{2} \log ^{2}\left(\frac{u^{p} \log ^{m}\left(u^{p}\right)}{\left\|u^{p} \log ^{m}\left(u^{p}\right)\right\|_{L^{2}}}\right) \\
& \leq \frac{1}{4}\left\|X\left(u^{p} \log m u^{p}\right)\right\|_{L^{2}}^{2}+C_{4}\left\|u^{p} \log ^{m} u^{p}\right\|_{L^{2}}^{2} \\
& \leq \frac{1}{2} \int_{\Omega}\left|X\left(u^{p}\right)\right|^{2} \log ^{2 m}\left(u^{p}\right)+m^{2} \int_{\Omega}\left|X\left(u^{p}\right)\right|^{2} \log ^{2 m-2}\left(u^{p}\right) \\
& +C_{4} \int_{\Omega} u^{2 p} \log ^{2 m}\left(u^{p}\right) \\
& \leq \frac{1}{2} \int_{\Omega}\left|X\left(u^{p}\right)\right|^{2} \log ^{2 m}\left(u^{p}\right)+\left(C_{4}+m^{2}\right) M_{1}^{2 m} P(m, p)(m !)^{2} .
\end{aligned}
$$


Sum up the three parts above, we get

$$
\begin{aligned}
& \int_{\Omega} u^{2 p} \log ^{2 m+2}\left(u^{p}\right) \leq \frac{1}{2} \int_{\Omega}\left|X\left(u^{p}\right)\right|^{2} \log ^{2 m}\left(u^{p}\right)+|\Omega|((m+1) !)^{2} \\
& +\left[2 C_{2}+C_{4}+m^{2}+a_{\infty}^{2}+2 p b_{\infty}+2 p a_{\infty}\left|\log A_{0}\right|\right. \\
& \left.+(1+|\Omega|) g_{\infty} / A_{1}\right] M_{1}^{2 m} P(m, p)(m !)^{2} .
\end{aligned}
$$

which implies by (4.8),

$$
\begin{gathered}
\int_{\Omega} u^{2 p} \log ^{2 m+2}\left(u^{p}\right)+\int_{\Omega}\left|X\left(u^{p}\right)\right|^{2} \log ^{2 m}\left(u^{p}\right) \leq\left[2 \Omega+4 C_{2}+2 C_{4}+10\right. \\
\left.+6 a_{\infty}^{2}+8 b_{\infty}+8 a_{\infty}\left|\log A_{0}\right|+2 g_{\infty}(1+|\Omega|) / A_{1}\right] M_{1}^{2 m} P(m+1, p)((m+1) !)^{2} .
\end{gathered}
$$

Proposition 4.2 is proved.

Proposition 4.3. Under the hypotheses of Proposition 4.1, if for some $p_{0} \geq 1$ and $A_{0} \geq e^{12}$ we have

$$
\|u\|_{L^{2 p_{0}}} \leq A_{0}
$$

then for

$$
M_{1} \geq\left[2|\Omega|+4 C_{2}+2 C_{4}+10+6 a_{\infty}^{2}+8 b_{\infty}+8 a_{\infty} \log A_{0}+2 g_{\infty}(1+|\Omega|) / A_{1}\right]^{\frac{1}{2}},
$$

and $\delta=1 / 2 M_{1}$, we have

$$
\int_{\Omega} u^{2 p_{0}(1+\delta)} \leq A_{0}^{2 p_{0}(1+\delta)\left(1+\left(\frac{1}{p_{0}(1+\delta)}\right)^{\frac{1}{3}}\right)}
$$

Proof. For any $\delta>0$, the estimate (4.7) gives that

$$
\begin{aligned}
& \left(\int_{\Omega}\left|\bar{u}^{p_{0}(1+\delta)}\right|^{2} d x\right)^{\frac{1}{2}}=\left(\int_{\Omega}\left|\bar{u}^{p_{0}} \bar{u}^{\delta p_{0}}\right|^{2} d x\right)^{\frac{1}{2}}=\left(\int_{\Omega}\left|\bar{u}^{p_{0}} e^{\delta \log \left(\bar{u}^{p_{0}}\right)}\right|^{2} d x\right)^{\frac{1}{2}} \\
= & \left(\int_{\Omega}\left|\bar{u}^{p_{0}} \sum_{m=0}^{\infty} \frac{\left(\delta \log \left(\bar{u}^{p_{0}}\right)\right)^{m}}{m !}\right|^{2} d x\right)^{\frac{1}{2}} \leq \sum_{m=0}^{\infty}\left(\int_{\Omega} \bar{u}^{2 p_{0}} \frac{\left(\delta \log \left(\bar{u}^{p_{0}}\right)\right)^{2 m}}{(m !)^{2}} d x\right)^{\frac{1}{2}} \\
\leq & \sum_{m=0}^{\infty} \frac{\delta^{m}}{m !}\left(\int_{\Omega} \bar{u}^{2 p_{0}} \log ^{2 m}\left(\bar{u}^{p_{0}}\right) d x\right)^{\frac{1}{2}} \leq \sum_{m=0}^{\infty} \delta^{m} M_{1}^{m} P\left(m, p_{0}\right) \leq p_{0}^{\sqrt{p_{0}}} \sum_{m=0}^{\infty}\left(\delta M_{1}\right)^{m} .
\end{aligned}
$$

For $\delta=1 / 2 M_{1}$, we have finally

$$
\int_{\Omega} u^{2 p_{0}(1+\delta)} d x \leq 4 p_{0}^{2 \sqrt{p_{0}}} A_{0}^{2 p_{0}(1+\delta)}
$$

Since for any $p_{0}>1$,

$$
4 p_{0}^{2 \sqrt{p_{0}}}=4 e^{2 \sqrt{p_{0}} \log p_{0}} \leq\left(e^{12}\right)^{2 p_{0}^{\frac{2}{3}}},
$$


which implies the estimate (4.10) if $A_{0} \geq e^{12}$.

We set now for $k \in \mathbb{N}$,

$$
p_{k}=p_{0}(1+\delta)^{k}, \quad A_{k}=A_{0}^{1+p_{0}^{-1 / 3} \sum_{j=1}^{k}\left(\frac{1}{1+\delta}\right)^{j / 3}},
$$

then Proposition 4.3 implies that

$$
\begin{aligned}
\int_{\Omega} u^{2 p_{0}(1+\delta)^{k+1}} & =\int_{\Omega} u^{2 p_{k}(1+\delta)} \leq A_{k}^{2 p_{k}(1+\delta)\left(1+\left(\frac{1}{p_{k}(1+\delta)}\right)^{1 / 3}\right)} \\
& \leq A_{0}^{2 p_{0}(1+\delta)^{k+1}\left(1+p_{0}^{-1 / 3} \sum_{j=1}^{k+1}\left(\frac{1}{1+\delta}\right)^{j / 3}\right)}
\end{aligned}
$$

where $\delta=\frac{1}{2} M_{1}$ and

$$
M_{1} \geq\left[2|\Omega|+4 C_{2}+2 C_{4}+10+6 a_{\infty}^{2}+8 b_{\infty}+8 a_{\infty}\left|\log A_{k}\right|+2 g_{\infty}(1+|\Omega|) / A_{1}\right]^{1 / 2} .
$$

We have now for $\delta=\frac{1}{2} M_{1} \leq 1 / 4$,

$$
\begin{aligned}
\frac{\log A_{k}}{\log A_{0}} & =1+p_{0}^{-1 / 3} \sum_{j=1}^{k}\left(\frac{1}{1+\delta}\right)^{j / 3} \leq 1+p_{0}^{-1 / 3} \sum_{j=1}^{\infty}\left(\frac{1}{1+\delta}\right)^{j / 3} \\
& =1+p_{0}^{-1 / 3} \frac{\left(\frac{1}{1+\delta}\right)^{1 / 3}}{1-\left(\frac{1}{1+\delta}\right)^{1 / 3}} \leq 1+4 p_{0}^{-1 / 3} M_{1} \leq 5 M_{1},
\end{aligned}
$$

where $M_{1}$ is independent of $k$, thus we have proved for any $k \in \mathbb{N}$,

$$
\int_{\Omega} u^{2 p_{0}(1+\delta)^{k}} \leq\left(A_{0}^{5 M_{1}}\right)^{2 p_{0}(1+\delta)^{k}} .
$$

If we choose $A_{0}=e^{12}$, then the estimate (4.2) holds for $\bar{A}=e^{60 M_{1}}$.

The regularity of the solution for the problem (1.5) and (1.6) can be deduced by following result:

Proposition 4.4. Suppose $a(x), b(x), g(x) \in C^{\infty}(\Omega)$, and there exist $a_{0}, b_{0}, g_{0}>$ 0, such that $a(x) \geq a_{0}, b(x) \geq b_{0}, g(x) \geq g_{0}$ in $\Omega$. Let $u \in H_{X, 0}^{1}(\Omega), u \geq 0,\|u\|_{L^{2}} \neq$ 0 be a weak solution of the problem (1.5) and (1.6), and $\partial \Omega$ is non characteristic. Then $u \in C^{\infty}(\Omega \backslash \Gamma) \bigcap C^{0}(\bar{\Omega} \backslash \Gamma)$, and $u(x)>0$ for all $x \in \Omega \backslash \Gamma$.

Proof. Suppose $x_{0} \in \Omega \backslash \Gamma$, then there exists a neighborhood $V_{0} \subset \Omega \backslash \Gamma$ of $x_{0}$, for $\varphi \in C_{0}^{\infty}\left(V_{0}\right)$ we shall prove that $v=\varphi u \in C^{\infty}\left(V_{0}\right)$. It follows from equation (1.5) that,

$$
-\Delta_{X} v=a(x) \varphi u \log u+b(x) \varphi u+g(x) \varphi+\sum_{j=1}^{m} \varphi_{j} X_{j} u+\varphi_{0} u=f_{0}+\sum_{j=1}^{m} X_{j} f_{j}
$$


with $\varphi_{j} \in C^{\infty}\left(V_{0}\right), f_{j} \in L^{\infty}\left(V_{0}\right), j=0, \ldots \ldots, m$. Since the system of vector fields $X$ satisfies the finitely type Hörmander's condition on $V_{0}$, the regularity result of [8] (see also $[7,9]$ ) implies that $u \in C^{\epsilon}\left(V_{0}\right)$ for some $\varepsilon>0$. If we have $u(x) \geq \alpha>0$ for $x \in V_{0}$, then by $t \log t \in C^{\infty}(t \geq \alpha)$, we can deduce $u \log u \in C^{\varepsilon}\left(V_{0}\right)$, thus we can prove by recurrence that $u \in C^{\infty}\left(V_{0}\right)$. For $x_{0} \in \partial \Omega \backslash \Gamma$, we have also $u \in C^{\epsilon}\left(V_{0} \bigcap \bar{\Omega}\right)$, but we know only $u \log u \in C^{0}\left(V_{0} \cap \bar{\Omega}\right)$, so we can not obtain the $C^{\infty}$ regularity of $u$ near to the boundary $\partial \Omega$. Therefore the Proposition 4.4 will be deduced by the following Lemma directly.

Lemma 4.5. Suppose $a(x), b(x), g(x)$ satisfy the conditions of Proposition 4.4, and $u \in C^{0}\left(\Omega_{1}\right), u \geq 0$ is a non trivial weak solution of the equation (1.5) on an open set $\Omega_{1} \subset \Omega$, then $u(x)>0$ for all $x \in \Omega_{1}$.

Proof. Suppose that $u\left(x_{0}\right)=0$ for some $x_{0} \in \Omega_{1}$, then for any $\epsilon>0$, there exists a small neighborhood $U_{0} \subset \Omega_{1}$ of $x_{0}$ such that $0 \leq u(x) \leq \epsilon$ on $\bar{U}_{0}$. Since $g(x)$ is continuous on $\bar{U}_{0}$, there exists $\alpha>0$ such that $g(x) \geq \alpha$ on $\bar{U}_{0}$.

Choosing $\epsilon$ small enough such that in $U_{0}$, we have

$$
a(x) u \log u+b(x) u<0
$$

and

$$
a(x) u \log u+b(x) u+g(x) \geq 0 .
$$

That is $\Delta_{X} u \leq 0$ in $U_{0}$. But $x_{0}$ is a minimum point of $u$, the maximum principle of Bony [10] implies that $u \equiv 0$ in $U_{0}$. That means $u$ is a trivial solution by continuous of $u$ in $\Omega_{1}$.

Acknowledgements The authors thank Prof. Wenyi Chen and Prof. Chaojiang $\mathrm{Xu}$ for their helpful conversations. The final version of the paper was done when $\mathrm{CH}$ was a visiting Professor in the Math. Inst. of Potsdam Univ., Germany during August, 2006, he would like to thanks Prof. B.-W. Schulze for the invitation and facilities.

\section{References}

[1] J.M. Bony, Principe du maximum, inégalité de Harnack et unicité du probléme de Cauchy pour les opérateurs elliptiques dégénérées, Ann. Inst. Fourier, 19, 1969, 227-304.

[2] A. Bahri and H. Berestycki, A perturbation method in critical point theory and applications, Trans. Amer. Math. Soc. 267, 1981, 1-32. 
[3] H. Brezis and L. Nirenberg, Positive solutions of nonlinear elliptic equations involving critical Sobolev exponent, Comm. Pure Appl. Math. 36, 1983, 437-477.

[4] M. Christ, Hypoellipticity in the infinitely degenerate regime, to appear in proceedings of Ohio State university conference on several complex variable.

[5] I. Ekeland, Nonconvex minimization problems, Bull. Amer. Math. Soc. 1, 1979, 443-473.

[6] M. Koike, A note on hypoellipticity for degenerate elliptic operates, Publ. RIMS Kyoto Univ., 27, 1991, 995-1000.

[7] J.J. Kohn, Hypoellipticity at points of infinite type, Analysis, geometry, number theory: the mathmatics of Leon Ehrenpreis (Philadelphia, 1998) 393-398, Contemp. Math., 251, 2000.

[8] Y. Morimoto and T. Morika, The positivity of Schrödinger operators and the hypoellipticity of second order degenerate elliptic operators, Bull. Sc. Math. 121, 1997, 507-547.

[9] Y. Morimoto and T. Morika, Hypoellipticity for elliptic operators with infinite degeneracy, "Partial Differential Equations and Their Applications" (Chen Hua and L. Rodino, eds.) World Sci. Publishing, River Edge, NJ, 1999, 240-259.

[10] Y. Morimoto and C. J. Xu, Logarithmic sobolev inequality and semi-linear Dirichlet problems for infinitely degenerate elliptic operators, Astérisque 234, 2003, 245-264.

[11] C. J. Xu, Subellptic variational problems, Bull. Soc. Math. France, 118, 1990, 147-169.

[12] C. J. Xu, Regularity problem for quasi-linear second order subellptic equations, Comm. Pure Appl. Math., 45, 1992, 77-96.

[13] C. J. Xu, Semilinear subelliptic equations and Sobolev inequality for vector fields satisfying Hörmander's condition, Chinese J. Contemp. Math., 15, 1994, 183-193. 